
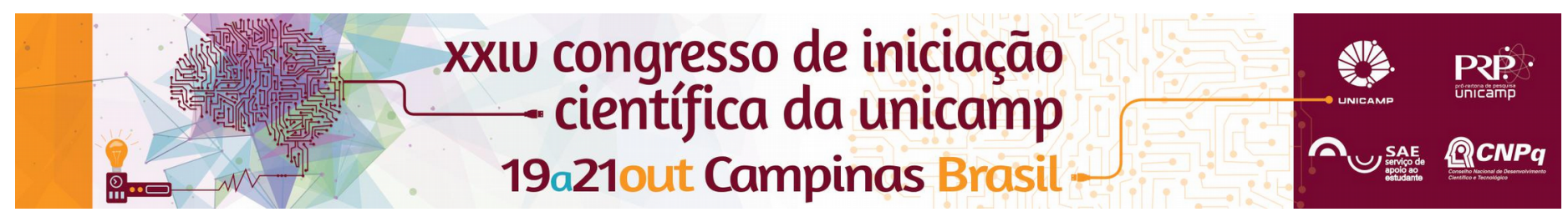

\title{
Dinâmica Simbólica: uma Introdução via Exemplos Hiperbólicos
}

\section{Pedro G. Mattos*, Régis Varão.}

\section{Resumo}

Este trabalho de Iniciação Científica tem como objetivo estudar alguns exemplos clássicos de sistemas dinâmicos e conhecer, através deles, os principais conceitos da área. Os exemplos estudados incluem os principais modelos dinâmicos, como os shifts de Bernoulli, endomorfismos expansores no círculo $\mathrm{S}^{1}$, automorfismos hiperbólicos no toro e a ferradura de Smale. Também são estudadas conjugações desses sistemas com o shift de Bernoulli e com cadeias de Markov.

\section{Palavras-chave:}

Sistemas dinâmicos, dinâmica simbólica, automorfismos hiperbólicos.

\section{Introdução}

A noção de sistemas dinâmicos utilizada foi a de um sistema dinâmico discreto: um par $(X, f)$ em que $X$ é um conjunto e $f: X \rightarrow X$ a dinâmica. Inicialmente, são estudados alguns sistemas dinâmicos clássicos. O estudo desses sistemas clássicos consiste na definição do espaço e da dinâmica envolvidos e de proposições e teoremas sobre periodicidade da órbita dos pontos, densidade de órbitas e de conjuntos de pontos no espaço em questão. Foram então estudados os conceitos de uma conjugação entre sistemas dinâmicos, o importante shift de Bernoulli e, por fim, algumas dinâmicas mais interessantes, como automorfismos hiperbólicos no toro $\mathbb{T}^{2}$, a ferradura de Smale e cadeias de Markov.

\section{Resultados e Discussão}

Os primeiros sistemas estudados foram a rotação e a expansão no cículo $S^{1}$, que é definido como o intervalo $[0,1]$ em que 0 e 1 são identificados. A rotação $R_{\alpha}$ é definida a partir de um número real $\alpha$ como $R_{\alpha}(x)=x+\alpha \bmod 1$. A expansão $E_{m}$ é definida a partir de um número natural $m$ como $E_{m}(x)=m x \bmod 1$. Pontos com órbitas periódicas são classificados.

Define-se uma conjugação entre dois sistemas dinâmicos $(X, f)$ e $(Y, g)$ como uma função sobrejetiva e contínua $\pi: X \rightarrow Y$ que satisfaz $\pi \circ f=g \circ \pi$ e cuja inversa também tem as mesmas propriedades.

Em seguida, o shift de Bernoulli foi estudado, sistema importante na teoria de sistemas dinâmicos por representar uma grande classe de sistemas. Ele consiste em um espaço de sequências $\Sigma_{m}=\{0, \ldots, m-1\}^{\mathbb{N}}$ e uma função $\sigma: \Sigma_{m} \rightarrow \Sigma_{m}$ definida por $\sigma\left(x_{n}\right)=\left(x_{n+1}\right)$. Algumas conjugações entre o shift de Bernoulli e sistemas estudados foram apresentadas.

O toro $\mathbb{T}^{2}$ é definido como o produto cartesiano de dois círculos $S^{1}$. Nesse espaço, são estudados automorfismos hiperbólicos definidos por uma matriz $A_{2 \times 2}$ que satisfaz três propriedades: (1) suas entradas são inteiras, (2) seu determinante é unitário e (3) os autovalores são distintos. A dinâmica leva um ponto $x$ do toro no ponto $A x$. A primeira propriedade garante que a dinâmica é bem definida no toro, a segunda, que ela é invertível, e a terceira, que ela é hiperbólica. São estudados pontos periódicos e conjuntos densos no toro relacionados a essa dinâmica.

Por fim, sistemas um pouco mais interessantes como a ferradura de Smale e cadeias de Markov foram brevemente estudados.

\section{Conclusão}

O estudo ainda não foi concluído. Nos primos meses, os sistemas citados no final da discussão serão mais aprofundadamente analisados em relação com conjugação com o shift de Bernoulli. Até o momento, o trabalho cumpriu o objetivo de apresentação da área de sistemas dinâmicos e permitiu que alguns problemas específicos fossem estudados e resolvidos. As referências usadas estão mencionadas abaixo.

\section{Agradecimentos}

Gostaria de agradecer ao meu orientador, José Régis Azevedo Varão Filho, e ao IMECC por tornarem esse trabalho possível. Este projeto foi financiado pela FAPESP.

\footnotetext{
${ }^{1}$ Brin,Michael; Stuck, Garrett. Introduction to dynamical systems. Cambridge University Press, Cambridge, 2002.

2 Barreira, Luis; Valls, Claudia. Dynamical systems. An introduction. Universitext. Springer, London, 2013.

${ }^{3}$ Devaney, Robert L. An introduction to chaotic dynamical systems. Algumas conjugações entre o shift de Bernoulli e sistemas estudados foram apresentadas. Second edition. Addison-Wesley Studies in Nonlinearity. Addison-Wesley Publishing Company, Advanced Book Program, Redwood City, CA, 1989.
} 